\title{
VOW: VARIANCE-OPTIMAL WAVELETS FOR THE STEERABLE PYRAMID
}

\author{
Pedram Pad, Virginie Uhlmann, and Michael Unser
}

\author{
Biomedical Imaging Group (BIG), \\ École polytechnique fédérale de Lausanne (EPFL), Switzerland.
}

\begin{abstract}
We study the issue of localization in the context of isotropic wavelet frames. We define a variance-type measure of localization and propose an algorithm based on calculus of variations to minimize this criterion under the constraint of a tight wavelet frame. Based on these calculations, we design the variance-optimal wavelet (VOW). Finally, we demonstrate the advantage of better localization in a practical image-processing task.
\end{abstract}

Index Terms - Isotropic wavelets, steerable pyramid, localization.

\section{INTRODUCTION}

Isotropic wavelets allow for a multi resolution decomposition of images which is orientation-free, while keeping all the desirable features of traditional wavelet bases. The resulting transformations hence benefit from good stability, approximation-theoretic properties, and fast implementation, among others. A directional ingredient can then be incorporated in order to rotate the wavelets and build a steerable pyramid [1, 2], which finds applications in many imageprocessing problems.

The basis functions generating such wavelets are required to be isotropic and to achieve a perfect reconstruction of the image. These conditions can be fulfilled by selecting a mother wavelet that is radially band-limited and satisfies some frequency-domain constraints [3, 4]. The most commonly used profile is Simoncelli's wavelet, as implemented in the classical version of the steerable pyramid [1]. Its design is inspired by biological vision [1] and has nice performance in a wide class of applications. Other available isotropic designs are the Meyer [5] and Papadakis wavelets [6].

The localization properties for isotropic wavelets have not been fully investigated yet. Localization is important from both theoretic and practical points of view. The theory dictates that more localized wavelets tend to decouple and sparsify signals more efficiently [7]. Also in practice, wavelets

This work was funded by the European Research Council under Grant ERC-2010-AdG 267439-FUN-SP and by the Swiss National Science Foundation under Grants 200020-144355 and 200020-121763. with better localization result in lesser oscillations and fewer truncation artifacts. We propose to address localization in the present paper.

We measure the localization of isotropic wavelets through a new criterion based on some normalized version of their variance. Then, we optimize the wavelet profile based on this measure. Guided by the outcome of this optimization, we introduce a new wavelet with a relatively simple mathematical expression which is nearly optimal. To demonstrate the benefit of an optimized design, we present examples of image reconstruction from edges in the wavelet domain. The underlying reconstruction task is formulated as a constrained optimization problem and solved iteratively. We observe that our optimized wavelet outperforms the previous ones in both PSNR and visual senses.

The paper is organized as follows: In Section 2, we recall the theoretical constraints and practical aspects related to the design of isotropic wavelet frames. In Section 3, we propose our measure of localization, the algorithm to optimize it, and, finally, the designed wavelet based on the outcome of the algorithm. Section 4 is devoted to the comparative evaluation of the proposed wavelets in practical applications.

\section{PRELIMINARIES ON THEORY AND IMPLEMENTATION OF BAND-LIMITED ISOTROPIC WAVELETS}

Let $\psi$ be a two-dimensional primal wavelet that generates the tight wavelet frame

$$
\psi_{i, \mathbf{k}}(\mathbf{x})=2^{-\frac{i}{2}} \psi\left(\frac{\mathbf{x}}{2^{i}}-\mathbf{k}\right)
$$

Because of isotropy, we have that $\psi(\mathbf{x})=\psi(\|\mathbf{x}\|)$, which is denoted by $\psi(r)$ for short. According to the properties of the Fourier transform, we know that the Fourier transform $h$ of $\psi$ is also isotropic. We can hence specify it by $h(\omega)$, where $\omega=$ $\|\boldsymbol{\omega}\|$. The radial wavelet is therefore the Hankel transform of $h$

$$
\psi(r)=\mathcal{H}\{h\}(r)=\int_{0}^{\infty} h(\omega) J_{0}(r \omega) \omega \mathrm{d} \omega .
$$


Now, let $N$ be a natural number and let $h$ satisfy the following constraints $[3,4]$ :

$$
\begin{array}{rlrl}
h(\omega)=0 & \text { for } \quad \omega>\pi, \\
\sum_{i \in \mathbb{Z}}\left|h\left(2^{i} \omega\right)\right|^{2}=1 & \text { for } \quad \omega \in \mathbb{R}^{+}, \\
\left.\frac{\mathrm{d}^{n}}{\mathrm{~d} \omega^{n}} h(\omega)\right|_{\omega=0}=0 & \text { for } & n=0, \ldots, N .
\end{array}
$$

It can be shown that the band-limited wavelet frame defined by (1) is tight, which is equivalent to the perfectreconstruction property.

From a practical point of view, the implementation of a wavelet transform as a filter-bank requires the definition of a pair of low- and high-pass filters, $h_{L}$ and $h_{H}$, corresponding to $h$. In this paper, we focus on wavelets with $h$ supported on $\left[\frac{\pi}{4}, \pi\right]$, for which the filters are described by

$$
\begin{aligned}
& h_{H}(\omega)= \begin{cases}h(\omega), & \omega<\frac{\pi}{2} \\
1, & \omega \geq \frac{\pi}{2}\end{cases} \\
& h_{L}(\omega)= \begin{cases}1, & \omega<\frac{\pi}{4} \\
h(2 \omega), & \omega \geq \frac{\pi}{4}\end{cases}
\end{aligned}
$$

Band-limited wavelet decompositions such as those of Simoncelli [1], Meyer [5], and Papadakis [6] lie in this category.

\section{LOCALIZED ISOTROPIC WAVELETS}

We first define a measure to quantify the localization of an isotropic wavelet. Then, using the calculus of variations, we find the gradient of this measure in the Hilbert space of isotropic wavelet profiles subject to Condition (4). Using the derived gradient, we propose a steepest-descent algorithm to find the most localized wavelet. We finally proceed with numerical optimization.

\subsection{Measure of Localization}

The measure of localization that we are going to use is a variance-type criterion for the primal wavelet profile. Since the properties of isotropic wavelets are given in terms of the corresponding $h$ function, we write the criterion as

$$
V\{h\}=\frac{\int_{0}^{\infty} r^{2}|\mathcal{H}\{h\}(r)|^{2} \mathrm{~d} r}{\int_{0}^{\infty}|\mathcal{H}\{h\}(r)|^{2} \mathrm{~d} r},
$$

where $\mathcal{H}\{h\}(r)$ is as in (2). This criterion quantifies how much the wavelet is concentrated around the origin. Small values of $V$ hence indicate better localization. Therefore, we would like to find the minimizer of $V$.

\subsection{Optimization Algorithm}

Since the functional $V$ is Fréchet-differentiable, we invoke Riesz' theorem to show that its infinite-dimensional gradient is well-defined. This suggests using a gradient-descent algorithm to minimize it. To simplify the manipulations, let us write $V$ as

$$
V\{h\}=\frac{A_{2}\{h\}}{A_{0}\{h\}},
$$

where

$$
A_{m}\{h\}=\int_{0}^{\infty} r^{m}|\mathcal{H}\{h\}(r)|^{2} \mathrm{~d} r .
$$

Now, we have that

$$
\nabla V\{h\}=\frac{\nabla A_{2}\{h\}}{A_{0}\{h\}}-\frac{\nabla A_{0}\{h\}}{A_{0}\{h\}} \frac{A_{2}\{h\}}{A_{0}\{h\}},
$$

so that we only need to determine the infinite-dimensional gradient of $A_{m}$ for $m=0,2$.

To that end, we first calculate the Gâteaux derivative of $A_{m}$ in the direction of a given function $g$ as

$$
\begin{aligned}
\mathrm{D}_{g} A_{m}\{h\} & =\lim _{\epsilon \rightarrow 0} \frac{\mathrm{V}\{h+\epsilon g\}-\mathrm{V}\{h\}}{\epsilon} \\
& =2 \int_{0}^{\infty} r^{m} \mathcal{H}\{h\}(r) \mathcal{H}\{g\}(r) \mathrm{d} r \\
& =2 \int_{0}^{\infty} r^{m} \mathcal{H}\{h\}(r)\left(\int_{0}^{\infty} g(\omega) \omega J_{0}(\omega r) \mathrm{d} \omega\right) \mathrm{d} r \\
& =2 \int_{0}^{\infty} g(\omega) \omega\left(\int_{0}^{\infty} r^{m} \mathcal{H}\{h\}(r) J_{0}(\omega r) \mathrm{d} r\right) \mathrm{d} \omega
\end{aligned}
$$

Thus, we get

$$
\nabla A_{m}\{h\}(\omega)=\omega \int_{0}^{\infty} r^{m} \mathcal{H}\{h\}(r) J_{0}(\omega r) \mathrm{d} r,
$$

which is then plugged into (11) to yield the gradient of $V$. Now, by going toward the opposite direction of the gradient of $V$, we can try to find its minimizer. We, however, have to be careful to stay on the manifold of band-limited isotropic wavelets satisfying Constraints (3)-(5).

According to the explanations in Section 2, we only consider wavelets supported on $\left[\frac{\pi}{4}, \pi\right]$ that automatically satisfy (3) and (5). To enforce (4) after each step, we project the obtained profile onto the manifold of profiles that satisfy (4). By simple manipulation, we deduce the projection map

$$
\mathcal{P}\{h\}(\omega)= \begin{cases}\sqrt{h(\omega)^{2}+h(2 \omega)^{2}} & \frac{\pi}{4}<\omega \leq \frac{\pi}{2} \\ \sqrt{h\left(\frac{\omega}{2}\right)^{2}+h(\omega)^{2}} & \frac{\pi}{2}<\omega \leq \pi\end{cases}
$$

for the functions $h$ supported on $\left[\frac{\pi}{4}, \pi\right]$.

Accordingly, the algorithm can be defined as 


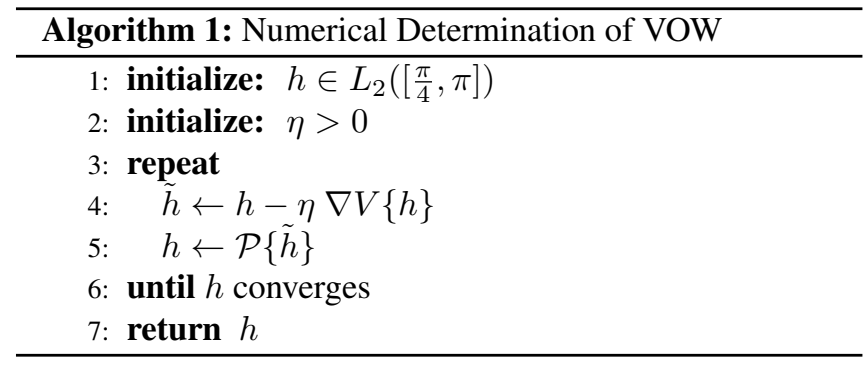

\subsection{Numerical Optimization}

To implement Algorithm 1, we take 512 samples of $h(\omega)$ with $\omega$ from $\pi / 4$ to $\pi$ uniformly on a logarithmic scale, hence simplifying the computation of the projection map (14). To calculate $\mathcal{H}\{h\}(r)$, we take the integral (2) from 0 to 100 with the trapezoid method using 1,000 intervals. The algorithm is run until the variations of $V\{h\}$ fall under $10^{-3}$. With this optimization procedure, we obtain the minimum value of 0.39 for $V\{h\}$. The resulting wavelet filter is depicted in Figure 1. The ringing effect observed is due to the Gibbs phenomenon that is the result of the truncation of the Fourier transform.

\subsection{Near-Optimal Wavelets with Closed-Form Formula}

To produce a usable wavelet profile, a closed-form formula is needed to easily implement a multi scale algorithm. We thus chose to fit some curves to the wavelet obtained numerically. We suggest an approximation that makes use of trigonometric tangent functions like in

$$
h(\omega)= \begin{cases}\sqrt{\frac{1}{2}+\frac{\tan \left(\kappa\left(1+2 \log _{2} \frac{2 \omega}{\pi}\right)\right)}{2 \tan (\kappa)}}, & \omega \in\left[\frac{\pi}{4}, \frac{\pi}{2}[\right. \\ \sqrt{\frac{1}{2}-\frac{\tan \left(\kappa\left(1+2 \log _{2} \frac{\omega}{\pi}\right)\right)}{2 \tan (\kappa)}}, & \omega \in\left[\frac{\pi}{2}, \pi\right] \\ 0, & \text { otherwise }\end{cases}
$$

where $\kappa \in\left[0, \frac{\pi}{2}\right)$. The value of $\kappa$ is found to be 0.75 . The profile of this wavelet is superimposed in Figure 1. The corresponding value of $V$ is 0.40 , which is not significantly different from the optimal one. We thus call $h$ in (15) the varianceoptimal wavelet (VOW).

\section{PRACTICAL EXAMPLE}

Using a given isotropic wavelet framework, we extract a multi scale "edge map" containing only a small subset of the wavelet coefficients, following the idea of $[8,9]$. Using constrained optimization techniques, we then show that a good approximation of the original image can be reconstructed from this subset.

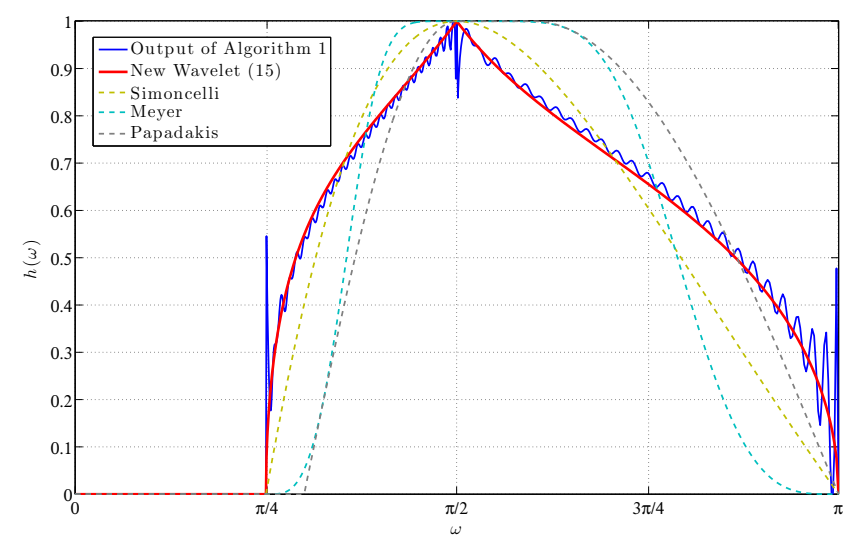

Fig. 1. Output of numerical optimization, fitted wavelet profile, and comparison with existing ones (Simoncelli, Meyer, and Papadakis).

\subsection{Image Reconstruction from Edges in the Wavelet Do- main}

Our goal translates to approximating the image using only coefficients corresponding to wavelet-based edges.

\subsubsection{Extraction of Wavelet-Based Edges}

The generalized Riesz wavelet transform presented in [10] allows one to easily generate gradient-like wavelets by specifying an appropriate shaping matrix. Based on that, a wavelet version of the standard Canny edge detector is implemented using the multi scale gradient signal while keeping the traditional Canny workflow, as proposed in [8]. As an output, we thus retain coefficients corresponding to singularities in the wavelet domain. All coefficients from the coarsest scale of the wavelet decomposition are kept so as to preserve the brightness information of the image.

\subsubsection{Image Reconstruction with Constrained Optimization}

As proposed in [9], we predicate our algorithm on the two following principles:

1. Wavelet-based edges must be conserved on the estimated image.

2. The estimated wavelet coefficients must project back onto an image while remaining as sparse as possible subject to Constraint 1 .

Reconstruction is therefore formulated as the constrained optimization problem (16), where $s_{0}$ is the original wavelettransformed image, and $\mathbf{k} \in S$ do index the locations of the wavelet-based edges. Thus, $S$ describes the image coordinates containing a non-zero value in the binary mask obtained in the edge-detection step. The sparsity constrain translates into the minimization of the $\ell_{1}$ norm of the wavelet transform 


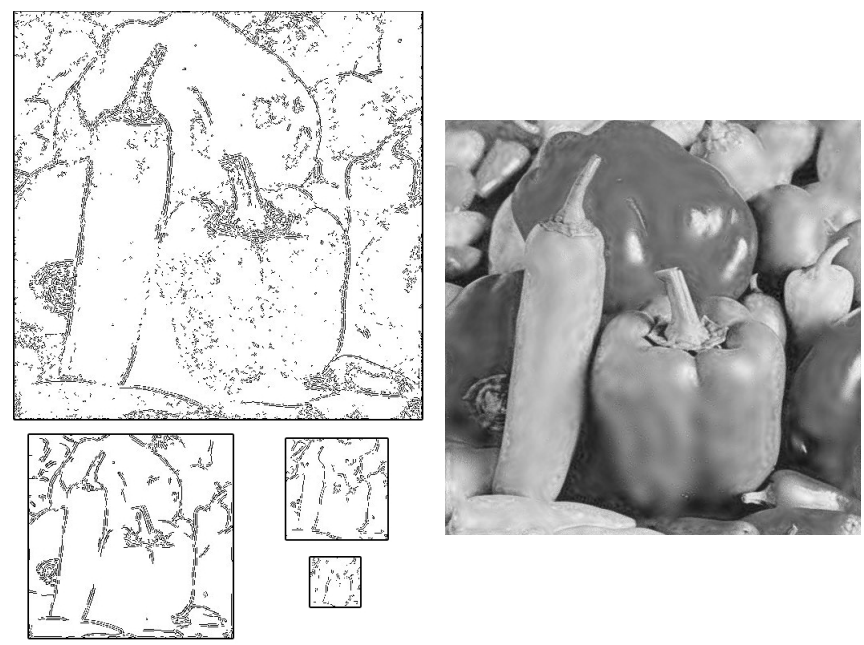

Fig. 2. Wavelet-based edge reconstruction of Peppers. Left: Binary mask featuring the wavelet coefficients saved for reconstruction at different scales (here, 4 scales were used). Right: Final result after reconstruction using VOW.

of the image. The optimization is finally constrained by the fact that we want to keep values of wavelet-based edges unchanged.

$$
\begin{aligned}
& \hat{\boldsymbol{f}}=\underset{\boldsymbol{f}}{\arg \min }\left\|\mathbf{W}^{H} \boldsymbol{f}\right\|_{1} \\
& \text { s. t. }\left[\mathbf{W}^{H} \boldsymbol{f}\right]_{\mathbf{k}}=\left[\boldsymbol{s}_{0}\right]_{\mathbf{k}}, \forall \mathbf{k} \in S
\end{aligned}
$$

We use alternating the direction method of multipliers (ADMM) [11] to solve the problem. We give in Figure 2 the reconstructed image as well as the coefficients retained by our multi scale wavelet-based edge detector for the Peppers test image.

\subsection{Results}

We investigate the reconstruction performance of our wavelets by comparing them to some of the most popular isotropic wavelet profiles, namely, Simoncelli [1], Papadakis [6], Meyer [5], and Shannon [2]. We evaluate results in terms of the PSNR of the reconstructed image. We fix the number of edge coefficients as the arbitrary value of $7.5 \%$ of
Table 1. Reconstruction from Wavelet-Based Edges

\begin{tabular}{ccccc}
\hline \hline Wavelet type & \multicolumn{3}{c}{ PSNR [dB] } & $V$ \\
& Peppers & Lena & Barbara & \\
\hline VOW (15) & $\mathbf{2 8 . 5 4}$ & $\mathbf{3 0 . 7 0}$ & $\mathbf{2 6 . 8 5}$ & $\mathbf{0 . 4 0}$ \\
Simoncelli [1] & 28.33 & 30.39 & 26.52 & 0.46 \\
Papadakis [6] & 27.65 & 29.46 & 26.39 & 0.49 \\
Meyer [5] & 27.03 & 29.31 & 25.81 & 0.67 \\
Shannon [2] & 25.77 & 27.99 & 25.66 & $+\infty$ \\
\hline
\end{tabular}

the total number of wavelet coefficients in the image for all experiments. All experiments are conducted with 4 scales of decomposition. The reconstruction results shown are obtained after 30 iterations of the ADMM algorithm.

As observed in Table 1, the VOW wavelet that we proposed in (15) outperforms the classical ones. Another interesting observation is that there exists a strong inverse relationship between $V$ and the PSNR. This confirms the relevance of our localization criterion. To allow for a visual comparison of the performance, we show in Figure 3 close-ups of the Barbara images reconstructed using different wavelets. Again, we observe that the result of the VOW is better than all the other popular wavelets we tested.

\section{CONCLUSIONS}

In this paper, we propose the VOW, a new kind of bandlimited isotropic wavelet. The VOW is designed by optimizing a variance-type measure and hence benefits from optimal localization. It was shown to outperform existing isotropic wavelets in the task of reconstructing an estimate of an image from a subset of its wavelet-based edges. Based on these results, we believe our localized isotropic wavelet to be an interesting candidate for image-processing tasks that involve steerable wavelets.

Our study highlights Simoncelli's wavelet as best second choice. We surmise that this is due to its degree of localization which, although suboptimal, is higher than that of the remaining classical isotropic wavelets we considered here. In addition to Simoncelli's biology-inspired construction, this observation can be used as a mathematical explanation for the efficiency of this wavelet in many different applications.
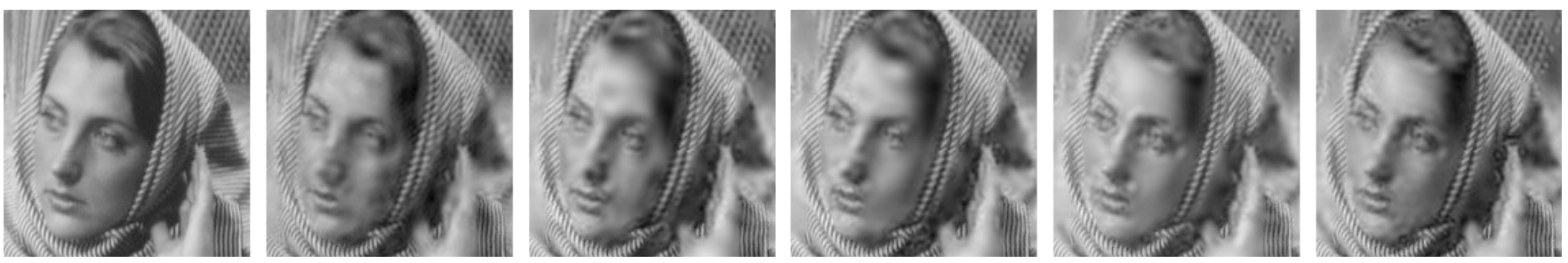

Fig. 3. Wavelet-based edge reconstruction of Barbara. From left to right: original image, Shannon, Meyer, Papadakis, Simoncelli, and VOW. 


\section{REFERENCES}

[1] J. Portilla and E.P. Simoncelli, "A parametric texture model based on joint statistics of complex wavelet coefficients," International Journal of Computer Vision, vol. 40, no. 1, pp. 49-70, October 2000.

[2] M. Unser, N. Chenouard, and D. Van De Ville, "Steerable pyramids and tight wavelet frames in $L_{2}\left(\mathbb{R}^{d}\right)$," IEEE Transactions on Image Processing, vol. 20, no. 10, pp. 2705-2721, October 2011.

[3] M. Papadakis, G. Gogoshin, I.A. Kakadiaris, D.J. Kouri, and D.K. Hoffman, "Nonseparable radial frame multiresolution analysis in multidimensions," Numerical Functional Analysis and Optimization, vol. 24, no. 7-8, pp. 907-928, 2003.

[4] S. Held, M. Storath, P. Massopust, and B. Forster, "Steerable wavelet frames based on the Riesz transform," IEEE Transactions on Image Processing, vol. 19, no. 3, pp. 653-667, March 2010.

[5] I. Daubechies, Ten lectures on wavelets. Philadelphia, PA: Society for Industrial and Applied Mathematics, 1992.

[6] J.R. Romero, S.K. Alexander, S. Baid, S. Jain, and M. Papadakis, "The geometry and the analytic properties of isotropic multiresolution analysis," Advances in Computational Mathematics, vol. 31, no. 1-3, pp. 283328, October 2009.

[7] P. Pad and M. Unser, "On the optimality of operatorlike wavelets for sparse AR(1) processes," in IEEE International Conference on Acoustics, Speech and Signal Processing (ICASSP), Vancouver, BC, May 26-31, 2013, pp. $5598-5602$.

[8] D. Van De Ville and M. Unser, "Complex wavelet bases, steerability, and the Marr-like pyramid," IEEE Transactions on Image Processing, vol. 17, no. 11, pp. 20632080, November 2008.

[9] N. Chenouard and M. Unser, "3D steerable wavelets in practice," IEEE Transactions on Image Processing, vol. 21, no. 11, pp. 4522-4533, November 2012.

[10] M. Unser and N. Chenouard, "A unifying parametric framework for 2D steerable wavelet transforms," SIAM Journal on Imaging Sciences, vol. 6, no. 1, pp. 102-135, January 2013.

[11] S. Boyd, N. Parikh, E. Chu, B. Peleato, and J. Eckstein, "Distributed optimization and statistical learning via the alternating direction method of multipliers," Foundations and Trends $\mathrm{R}$ in Machine Learning, vol. 3, no. 1, pp. 1-122, January 2011. 